\title{
Dynamical supersymmetry breaking in models with a Green-Schwarz mechanism
}

\author{
Nima Arkani-Hamed ${ }^{\mathrm{a}, 1}$, Michael Dine ${ }^{\mathrm{b}, \mathrm{c}}$, Stephen P. Martin ${ }^{\mathrm{b}, \mathrm{c}}$ \\ a Stanford Linear Accelerator Center, Stanford, CA 94309, USA \\ b Santa Cruz Institute for Particle Physics, Santa Cruz, CA 95064, USA \\ c Physics Department, University of California, Santa Cruz, CA 95064, USA \\ Received 7 April 1998 \\ Editor: M. Cvetič
}

\begin{abstract}
We consider supersymmetry breaking in theories with gaugino condensation in the presence of an anomalous U(1) symmetry with anomaly cancellation by the Green-Schwarz mechanism. In these models, a Fayet-Iliopoulos $D$-term can give important contributions to the soft supersymmetry-breaking scalar masses. Most discussions of this possibility have ignored the dilaton field. We argue that this is not appropriate in general, and show that the $F$-term contributions to the soft breaking terms are comparable to or much larger than the $D$-term contributions, depending on how the dilaton is stabilized. We discuss phenomenological implications of these results. (C) 1998 Published by Elsevier Science B.V. All rights reserved.
\end{abstract}

When considering supersymmetry breaking, one of the most serious issues is understanding the flavor structure of the soft supersymmetry breaking mass terms. There are several proposals to explain how a structure consistent with known facts about flavor violation might arise:

1. Universal soft scalar masses at some high energy scale. In the context of, say, a supergravity theory, such a proposal is a convenient starting point for phenomenology but is not, by itself, natural. It corresponds to arbitrarily imposing a relation among a very large number of parameters.

2. Dilaton domination. In string theory, if the $F$ term of the dilaton is the principle source of supersymmetry breaking, this leads to universal soft masses, provided one assumes that the Kähler potential for the dilaton is well-approximated by its weak coupling form. However, it is hard to understand how the dilaton potential can be stabilized unless there are large corrections to the Kähler potential.

3. Flavor symmetries. It is possible that approximate flavor symmetries can give squark and slepton degeneracy or alignment, while permitting the observed flavor violations among the fermions.

4. Low energy, gauge mediated supersymmetry breaking. In such schemes, gauge interactions serve as the principle messengers of supersymmetry breaking. Soft breaking masses are functions of gauge quantum numbers, providing adequate degeneracy to suppress flavor changing processes.

\footnotetext{
${ }^{1}$ Work supported by the Department of Energy under contract DE-AC03-76SF00515. 
The focus of this letter is a fifth suggestion:

5. Fayet-Iliopoulos D-term breaking as the source of soft scalar masses. This possibility has been widely discussed in the literature. As proposed in [1] and [2], this idea relies on the existence of a $U(1)_{X}$ gauge symmetry with anomaly cancellation implemented by a non-trivial transformation of the dilaton according to the Green-Schwarz mechanism [3]. After supersymmetry breaking and spontaneous breaking of $U(1)_{X}$, the corresponding $D$-term obtains a vacuum expectation value (VEV). Light fields carry a $U(1)_{X}$ charge, so the $D$-term VEV contributes to the soft squared masses of the squarks, sleptons and Higgs bosons of the Minimal Supersymmetric Standard Model (MSSM). If all of the squarks and sleptons carry the same $U(1)_{X}$ charge, this can lead to flavor-independent contributions to soft breakings. Alternatively, it might lead to interesting patterns of alignment, if the Yukawa couplings are correlated with the $U(1)$ charges in just the right way. These possibilities have been explored in recent model-building, including [4-11].

This last proposal seems quite exciting. It seems to relate a very microscopic phenomenon (the generation of a Fayet-Iliopoulos $D$-term through the Green-Schwarz mechanism) in a quite well-defined and controllable way to measurable properties of the low energy theory. But upon further consideration, the suggestion raises several puzzles.

First, one might wonder why $D$-terms should appear in the low energy theory, given that the $U(1)_{X}$ gauge symmetry is broken at a very high energy scale (one or two orders of magnitude below the Planck scale), well above the scale of supersymmetry breaking. Indeed, these terms can be understood from a low energy viewpoint as arising from integrating out the corresponding massive vector supermultiplet. This gives rise to corrections to the Kähler potential for the light fields, which in turn contribute to the low energy soft breakings. To see this, consider first a general model with canonical Kähler potential terms for some chiral superfields $\phi_{i}$ with $U(1)_{X}$ charges $q_{i}$. Then the scalar potential is

$$
V=\sum_{i}\left|\frac{\partial W}{\partial \phi_{i}}\right|^{2}-\frac{1}{2 g_{X}^{2}} D_{X}^{2}-D_{X}\left(\sum_{i} q_{i}\left|\phi_{i}\right|^{2}+\xi^{2}\right),
$$

where $W$ is the superpotential and $\xi^{2}$ is a constant Fayet-Iliopoulos term in the lagrangian before symmetry breaking. In the low-energy theory there are contributions to the soft masses of the light fields arising from the expectation value of the $D$-term:

$$
m_{\phi_{i}}^{2}=-q_{i}\left\langle D_{X}\right\rangle
$$

To relate this to properties of the light fields, note first that at a stationary point of the potential the VEV of the $D$-term is related to the $F$ term VEVs according to

$$
\left\langle D_{X}\right\rangle=-\frac{g_{X}^{2}}{M_{X}^{2}} \sum_{i} q_{i}\left|\left\langle F_{i}\right\rangle\right|^{2},
$$

where $M_{X}^{2}=g_{X}^{2} \sum_{i} q_{i}^{2}\left|\left\langle\phi_{i}\right\rangle\right|^{2}$ is the (mass) ${ }^{2}$ of the $U(1)_{X}$ massive supermultiplet; this is easily shown using the gauge invariance of $W$. This corresponds to the fact that tree-level exchange of the heavy gauge multiplet gives a contribution to the low-energy Kähler potential:

$$
\Delta K=-\frac{g_{X}^{2}}{M_{X}^{2}} q_{i} q_{j} \phi^{* i} \phi_{i} \phi^{* j} \phi_{j}
$$

Now if we suppose that some of the $\phi_{i}$ 's have non-zero $F$ components, Eq. (2) again follows. 
Thinking about the problem in this way makes clear why one might hope that the $D$ term provides the dominant contribution to supersymmetry breaking. Since $U(1)_{X}$ is broken at scale one or two orders of magnitude lower than $M_{P}$, the $U(1)_{X}$ gauge boson mass is lighter than the Planck scale and so the (controllable) $D_{X}$ contributions to the soft masses $\sim 1 / M_{X}^{2}$ can dominate over the (uncontrollable) $\sim 1 / M_{P}^{2}$ supergravity contributions.

This way of thinking about the $D$ term suggests a strategy for model building with $U(1)_{X}$ serving as a "messenger" of supersymmetry breaking. One can consider a theory with a sector which breaks supersymmetry, such as the $(3,2)$ model [12], and gauge a $U(1)$ symmetry. The $F$ terms in the symmetry breaking sector then give rise to a modification of the Kähler potential for any other fields charged under the symmetry as in Eq. (4). The resulting pattern of symmetry breaking then depends on the charge assignments of the fields, and can produce interesting patterns of degeneracy or alignment. Such models, however, suffer from some phenomenological difficulties. Scalars not charged under $U(1)_{X}$ (which typically include the top squark) can only get soft masses from $1 / M_{P}$ effects and are therefore much lighter than the charged scalars. Because there are typically no low dimension, gauge invariant operators in theories of dynamical supersymmetry breaking, gaugino masses tend to be even further suppressed ${ }^{2}$. Apart from the usual fine-tuning problem with heavy scalars [13], this spectrum also typically drives the top squark squared mass negative at the weak scale [14].

In the above scenario, the anomalous $U(1)$ is not in itself involved in the dynamics of supersymmetry breaking. A more interesting possibility $[1,2]$ has $U(1)_{X}$ playing a crucial role in the supersymmetry breaking dynamics. Schematically, these models typically include a Standard Model singlet field $\varphi$, whose VEV and charge are appropriate to cancel the Fayet-Iliopoulos term. This field couples to some fields charged under a non-abelian group. Upon integrating out these fields, gaugino condensation in the low energy theory generates a dynamical superpotential for $\varphi$. The VEV of $\varphi$ needed to cancel the Fayet-Iliopoulos term is not at a stationary point of the superpotential, so supersymmetry is claimed to be broken.

In these models, it is customary to ignore the dilaton (essential for anomaly cancellation) and assume that it does not play an important role in supersymmetry breaking. But analyzed in this way, there is a puzzle: a massless goldstino does not appear in the spectrum. The $U(1)$ gaugino and the fermionic component of $\varphi$ acquire a Dirac mass from the Higgs mechanism, and there is no light fermion arising from the non-abelian dynamics. The absence of a goldstino is clearly connected with the anomaly. To see this, rather than considering the formal proof of the supersymmetric analog of Goldstone's theorem, consider instead the explicit realization of the theorem in weakly coupled theories with a canonical Kähler potential for all of the chiral fields. Then the fermion mass matrix has the form

$$
M_{\text {fermion }}=\left(\begin{array}{cc}
0 & \sqrt{2} g_{X} q_{i} \phi^{* i} \\
\sqrt{2} g_{X} q_{j} \phi^{* j} & \frac{\partial^{2} W}{\partial \phi_{i} \partial \phi_{j}}
\end{array}\right)
$$

in the (gaugino, chiral fermion) basis with canonical kinetic terms. Using the extremization condition for the scalar potential $\partial V / \partial \phi_{i}=0$, one finds that this matrix annihilates the eigenvector $\left(\left\langle D_{X}\right\rangle / \sqrt{2}, g_{X}\left\langle F_{i}\right\rangle\right)$ corresponding to the goldstino wavefunction, but only if the superpotential is gauge invariant.

\footnotetext{
${ }^{2}$ An exception occurs in models with singlets. However, in all such models, it is necessary to prohibit some couplings. This can only be done naturally by imposing symmetries, which invariably suppress gaugino masses.
} 
This immediately resolves the puzzle of the missing goldstino in these models. In these theories, the superpotential $W$ is generally not gauge invariant unless one maintains its explicit dependence on the dilaton chiral superfield $S$, which transforms under a $U(1)_{X}$ gauge transformation, $A_{\mu}^{X} \rightarrow A_{\mu}^{X}+\partial_{\mu} \alpha$, according to

$$
S \rightarrow S+i \frac{\delta_{\mathrm{GS}}}{2} \alpha,
$$

with

$$
\delta_{\mathrm{GS}}=\frac{1}{192 \pi^{2}} \sum_{i} q_{i}
$$

A typical non-perturbative superpotential has the form:

$$
W_{\mathrm{np}}=\phi^{A} e^{-p S / \delta_{\mathrm{GS}}} \text {, }
$$

where $p$ is a model-dependent positive number of order 1 , and gauge invariance requires that $\phi^{A}$ carries $U(1)_{X}$ charge $p / 2$. Now it is apparent that in order to properly describe spontaneous supersymmetry breaking with a massless goldstino, it is mandatory to include $S$ as a dynamical degree of freedom along with the matter fields. Indeed, the light degrees of freedom left after supersymmetry breaking and $U(1)_{X}$ breaking must include $S$, and the goldstino is predominantly the dilatino (the fermionic component of $S$ ). This means that the dilaton $F$-term, $\left(F_{S}\right)$, plays a crucial role in supersymmetry breaking and its contributions to the soft breaking terms in the low-energy theory cannot be neglected.

To understand this, one can consider the origin of the $D$-term in the low-energy theory with the heavy fields (including fields which transform under the strongly-coupled part of the gauge group) integrated out. Consider a model which includes a chiral superfield $\varphi$ with $U(1)_{X}$ charge -1 . In order to be gauge invariant, the Kähler potential for the dilaton must be a function of $S+S^{*}-\delta_{\mathrm{GS}} X$, where $X$ is the vector superfield for $U(1)_{X}$, so that

$$
K_{\mathrm{tot}}=e^{-2 X} \varphi^{*} \varphi+K\left(S+S^{*}-\delta_{\mathrm{GS}} X\right) .
$$

The $U(1)_{X} D$-term can now be written as

$$
D_{X}=-g_{X}^{2}\left(\xi^{2}-|\varphi|^{2}\right),
$$

where $\xi^{2}=-\delta_{\mathrm{GS}} K^{\prime} / 2>0$ is the Fayet-Iliopoulos term and $g_{X}^{2}=2 / k_{X}\left(S+S^{*}\right)$ with $k_{X}$ the Kac-Moody level for $U(1)_{X}$. Now, at the minimum of the potential $|\varphi|^{2}$ obtains a VEV which nearly cancels $\xi^{2}$. To relate this to the dilaton $F$-term $\mathrm{VEV}$, one can make a field redefinition, shifting $X \rightarrow X+\ln |\varphi|$ and $S \rightarrow S+\frac{\delta_{\mathrm{GS}}}{2} \ln \varphi$ to obtain the "unitary gauge" version of the Kähler potential:

$$
K_{\text {tot }}=e^{-2 X}+K\left(S+S^{*}-\delta_{\mathrm{GS}} X\right),
$$

in which the dependence on the absorbed field $\varphi$ has been eliminated. Now one can integrate out the massive vector supermultiplet $X$ using its equation of motion

$$
X=-\frac{1}{2} \ln \left(-\delta_{\mathrm{GS}} K^{\prime}\right) \text {. }
$$

(Here and in the following, a prime always means a derivative with respect to $S$.) Taking the $D$-term component of both sides yields

$$
D_{X}=-\left|F_{S}\right|^{2}\left(\ln K^{\prime}\right)^{\prime \prime}+\frac{\delta_{\mathrm{GS}}}{2} D_{X}\left(\ln K^{\prime}\right)^{\prime},
$$

so that at the minimum of the potential

$$
\left\langle D_{X}\right\rangle=\left|\left\langle F_{S}\right\rangle\right|^{2}\left(-\frac{K^{\prime \prime \prime}}{K^{\prime}}+\left(\frac{K^{\prime \prime}}{K^{\prime}}\right)^{2}\right)\left(1-\frac{\delta_{\mathrm{GS}} K^{\prime \prime}}{2 K^{\prime}}\right)^{-1} .
$$

Here $K$ is now taken to be a function of the scalar component of $\left\langle S+S^{*}\right\rangle$. This general formula relates the $U(1)_{X} D$-term to the dilaton $F$-term VEV and the derivatives of the Kähler potential. The latter are constrained, but not determined, by the requirement that the scalar potential is stable with respect to variations of the dilaton. 
If one were now to naively substitute the weak coupling form of the Kähler potential, $K=-\ln \left(S+S^{*}\right)$, into Eq. (14), then one might conclude that $\left\langle D_{X}\right\rangle=-\left|\left\langle F_{S}\right\rangle\right|^{2} /\left\langle S+S^{*}\right\rangle^{2}$ up to small corrections of order $\delta_{\mathrm{GS}} / 2\left\langle S+S^{*}\right\rangle$. This already suggests that the $D$ term is not more important than other contributions to the soft breakings. However, this Kähler potential is only appropriate for large $S$, but the true vacuum probably lies in a region where weak coupling is not valid. At the true minimum, this estimate may not be correct. For instance, if the dilaton is stabilized by Kähler potential corrections, the derivatives of $K$ cannot all be close to the weakly coupled prediction. To see this, note that the dilaton-dependent part of the scalar potential includes

$$
V=\left|W_{\mathrm{np}}^{\prime}\right|^{2} / K^{\prime \prime}+\ldots,
$$

where $W_{\mathrm{np}}$ is of the form given in Eq. (8). If this term dominates the contributions to the minimization condition $V^{\prime}=0$, then it follows that

$$
\frac{p}{\delta_{\mathrm{GS}}} K^{\prime \prime}=-K^{\prime \prime \prime}+\ldots,
$$

so that $K^{\prime \prime}$ must be parametrically suppressed by one power of $\delta_{\mathrm{GS}}$ compared to $K^{\prime \prime \prime}$ at the minimum of the potential. Of course if the dilaton is stabilized by corrections to the superpotential, the weak coupling estimate for $\left\langle D_{X}\right\rangle$ given above can be correct, so that $\left\langle D_{X}\right\rangle$ and $\left\langle F_{S}\right\rangle$ are comparable in size.

Despite recent progress in string theory, the mechanism for stabilizing the dilaton - if one exists - is not known. Various models have been proposed, including specially constructed superpotentials [15], and Kähler potentials motivated by non-perturbative string theory considerations [16-20]. For illustrative purposes, we also consider a Kähler potential which has a different structure but which for our present purposes contains the essential features of the latter class of models. Our model is quite simple, with a Kähler potential chosen to have the correct behavior at weak coupling, a small number of parameters, and a minimum of the desired sort. We take

$$
K=-\ln \left(S+S^{*}\right)-\frac{2 s_{0}}{S+S^{*}}+\frac{b+4 s_{0}^{2}}{6\left(S+S^{*}\right)^{2}},
$$

where $s_{0}$ and $b$ are non-negative constants. For large $S \gg s_{0}$, this agrees with the weak coupling result. Now

$$
K^{\prime \prime}=\frac{\left(S+S^{*}-2 s_{0}\right)^{2}+b}{\left(S+S^{*}\right)^{4}} .
$$

For $b>0$, this is positive-definite, as required for sensible kinetic terms. In the limit $b \rightarrow 0$, it has a zero at $S=s_{0}$. Therefore, the scalar potential Eq. (15) diverges at $S=s_{0}$ for $b \rightarrow 0$. In that case it is clear that the exponentially-falling superpotential pushes $S$ out to a local minimum just less than $s_{0}$. As long as we suppose ${ }^{3}$ that $b \leqq \delta_{\mathrm{GS}}^{2} / p^{2}$, there will be a stable local minimum near $S=s_{0}-\delta_{\mathrm{GS}} / p$. At that minimum, the derivatives of the Kähler potential are given by (to leading order in $\delta_{\mathrm{GS}}$ ):

$$
K^{\prime}=-\frac{1}{6 s_{0}} ; \quad K^{\prime \prime}=\frac{\delta_{\mathrm{GS}}^{2}}{4 p^{2} s_{0}^{4}} ; \quad K^{\prime \prime \prime}=-\frac{\delta_{\mathrm{GS}}}{4 p s_{0}^{4}} .
$$

So we see that $K^{\prime \prime \prime}$ and $K^{\prime \prime}$ are both parametrically suppressed, by $\delta_{\mathrm{GS}}$ and $\delta_{\mathrm{GS}}^{2}$ respectively. To leading order in $\delta_{\mathrm{GS}}$, we therefore find that

$$
\left\langle D_{X}\right\rangle=-\left|\left\langle F_{S}\right\rangle\right|^{2} \frac{K^{\prime \prime \prime}}{K^{\prime}}=-\frac{3 \delta_{\mathrm{GS}}}{2 p s_{0}^{3}}\left|\left\langle F_{S}\right\rangle\right|^{2} .
$$

In the general class of models where the dilaton is stabilized by a near vanishing of $K^{\prime \prime}$, we conclude that $\left\langle D_{X}\right\rangle$ is parametrically suppressed by $\delta_{\mathrm{GS}}$ relative to $\left\langle F_{S}\right\rangle$.

\footnotetext{
${ }^{3}$ This evidently entails a fine tuning.
} 
The Kähler potential of Eq. (17) should provide a useful toy model for dilaton stabilization in contexts other than that considered here. If one supposes that supersymmetry is hierarchically broken, there is an approximate moduli space, and it is presumably appropriate, even at strong coupling, to write an effective action for the light fields such as the dilaton. In the present context, however, some of the fields we are including in the effective lagrangian beneath the Planck scale (namely the $U(1)_{X}$ gauge field) approach Planck scale masses at strong coupling, and it is not clear that including them incorporates the correct dynamics. However, we believe the model above gives some qualitative indication of the correct physics, and at any rate, since the dilaton should ultimately be stabilized at moderate coupling, the gauge multiplet may be light enough to justifiably be included in the low energy theory.

If the dilaton is stabilized by some other means, it is possible to imagine that the suppression of $\left\langle D_{X}\right\rangle$ compared to $\left|\left\langle F_{S}\right\rangle\right|^{2}$ that we have just found does not hold, even though Eq. (16) is satisfied. This could be the case for example if $K^{\prime \prime \prime}$ is extremely large at some value of $S$, corresponding to a sudden change in $K^{\prime \prime}$. However, the nicest thing one can say about such a possibility is that it is not particularly appealing. As already mentioned, it is also possible to imagine that an unspecified superpotential effect stabilizes $S$. Even in that case, however, Eq. (14) implies that $\left\langle F_{S}\right\rangle$ is at least comparable to $\left\langle D_{X}\right\rangle$.

It is instructive to consider how the preceding discussion is realized in a concrete example, treating the gaugino condensation in the microscopic theory explicitly. We will consider the model proposed by Binétruy and Dudas in [1], with gaugino condensation from a gauged $S U\left(N_{c}\right)$ symmetry, and taking $N_{f}=1$ for simplicity. In addition to the $S U\left(N_{c}\right)$-singlet field $\varphi$ with $U(1)_{X}$ charge -1 , there are chiral superfields $Q$ and $\bar{Q}$ transforming under $S U\left(N_{c}\right) \times U(1)_{X}$ as $\left(\mathbf{N}_{c}, q\right)$ and $\left(\overline{\mathbf{N}}_{c}, \bar{q}\right)$ respectively. It is convenient to minimize the potential along the $S U\left(N_{c}\right)$-flat direction using the canonically normalized meson superfield $t=(2 Q \bar{Q})^{1 / 2}$, so that the Kähler potential is $t^{*} t\left(e^{2 q X}+e^{2 \bar{q} X}\right) / 2+\varphi^{*} \varphi e^{-2 X}+K\left(S+S^{*}-\delta_{\mathrm{GS}} X\right)$. Then the scalar potential is given by

$$
V=K^{\prime \prime}\left|F_{S}\right|^{2}+\left|\frac{\partial W}{\partial \varphi}\right|^{2}+\left|\frac{\partial W}{\partial t}\right|^{2}+\frac{1}{2 g_{X}^{2}} D_{X}^{2},
$$

where $F_{S}=-W^{\prime *} / K^{\prime \prime}$ and

$$
D_{X}=-g_{X}^{2}\left(\frac{q+\bar{q}}{2}|t|^{2}-|\varphi|^{2}+\xi^{2}\right) \text {. }
$$

The gauge-invariant superpotential is given by

$$
W=m \frac{t^{2}}{2}\left(\frac{\varphi}{M_{P}}\right)^{q+\bar{q}}+\left(N_{c}-1\right)\left(\frac{2 \Lambda^{3 N_{c}-1}}{t^{2}}\right)^{\frac{1}{N_{c}-1}}
$$

where the last term is the ADS superpotential [12] and corresponds to $W_{\mathrm{np}}$ in Eq. (8). The dynamical scale $\Lambda$ depends on the dilaton field according to

$$
\left(\frac{\Lambda}{M_{P}}\right)^{3 N_{c}-1}=e^{-8 \pi^{2} k_{N} S}=e^{-2(q+\bar{q}) S / \delta_{\mathrm{GS}}},
$$

where $k_{N}$ is the Kac-Moody level of the $S U\left(N_{c}\right)$ gauge group. The difference between the present treatment and [1] is that we will include the effects of the first term $K^{\prime \prime}\left|F_{S}\right|^{2}$ in the scalar potential Eq. (21). 
Now we can search for a local minimum of the scalar potential with respect to variations of $t$ and $\varphi$, in the neighborhood of $\langle\varphi\rangle=\xi$. Following [1], we can define convenient parameters

$$
\hat{m} \equiv m\left(\frac{\xi}{M_{P}}\right)^{q+\bar{q}} ; \quad \epsilon=\left(\frac{\Lambda}{\xi}\right)^{\frac{3 N_{c}-1}{N_{c}}}\left(\frac{\xi}{\hat{m}}\right)^{\frac{N_{c}-1}{N_{c}}},
$$

with $\epsilon \ll 1$. Then the location of the minimum and the auxiliary field VEVs can be determined as an expansion in $\epsilon$. One finds a local minimum at

$$
\begin{aligned}
& \left\langle\varphi^{2}\right\rangle=\xi^{2}[1+\epsilon(q+\bar{q})+\ldots] \\
& \left\langle t^{2}\right\rangle=2 \epsilon \xi^{2}\left[1+\epsilon(q+\bar{q})^{2} \frac{N_{c}-1}{2 N_{c}^{2}}\left(1-2 N_{c}-\frac{2 K^{\prime}}{\delta_{\mathrm{GS}} K^{\prime \prime}}\right)+\ldots\right] .
\end{aligned}
$$

At this minimum,

$$
\begin{aligned}
& \left\langle D_{X}\right\rangle=\epsilon^{2} \hat{m}^{2}(q+\bar{q})^{2}\left[1-\frac{(q+\bar{q})}{N_{c}}\left(1-\frac{2 K^{\prime}}{\delta_{\mathrm{GS}} K^{\prime \prime}}\right)\right], \\
& \left\langle F_{S}\right\rangle=\epsilon \hat{m}(q+\bar{q}) \frac{K^{\prime}}{K^{\prime \prime}},
\end{aligned}
$$

to the lowest non-trivial order in $\epsilon$. (The $F$-terms for $t$ and $\varphi$ also obtain VEVs, but they have a much smaller effect on the soft masses of the fields in the low-energy theory.) The terms in Eqs. (26)-(28) which do not explicitly involve $K^{\prime \prime}$ are the ones computed in [1]. However, they are actually suppressed compared to the terms which arise from including the $K^{\prime \prime}\left|F_{S}\right|^{2}$ term in the potential, at least for models where the dilaton is stabilized as described above.

Using Eqs. (28) and (29), it is now possible to compare the dominant sources of supersymmetry breaking:

$$
\left\langle D_{X}\right\rangle /\left.\left\langle F_{S}\right\rangle\right|^{2}=\frac{2(q+\bar{q})}{N_{c} \delta_{\mathrm{GS}}} \frac{K^{\prime \prime}}{K^{\prime}}\left(1-\frac{\delta_{\mathrm{GS}} K^{\prime \prime}}{2 K^{\prime}}\right)+\left(\frac{K^{\prime \prime}}{K^{\prime}}\right)^{2} .
$$

At first sight, this does not seem to agree with Eq. (14), since it does not even involve $K^{\prime \prime \prime}$. However, this is merely because we have not yet used the minimization condition for the dilaton, which in this model can be written as

$$
\frac{K^{\prime \prime \prime}}{K^{\prime}}=-\frac{2(q+\bar{q})}{N_{c} \delta_{\mathrm{GS}}} \frac{K^{\prime \prime}}{K^{\prime}}\left(1-\frac{\delta_{\mathrm{GS}} K^{\prime \prime}}{2 K^{\prime}}\right)^{2}+\frac{\delta_{\mathrm{GS}}}{2}\left(\frac{K^{\prime \prime}}{K^{\prime}}\right)^{3}
$$

to leading order in $\epsilon$. Using this one can show that Eq. (30) is precisely equivalent to Eq. (14). In particular, the $\delta_{\mathrm{GS}}$ in the denominator of the RHS of Eq. (30) does not necessarily imply any enhancement of $\left\langle D_{X}\right\rangle$; for example, in the model of dilaton stabilization we discussed above, $K^{\prime \prime}$ is expected to be parametrically suppressed by $\delta_{\mathrm{GS}}^{2}$.

As we discussed earlier, it is also possible to understand the presence of the $D$-term in the low energy theory as arising from integrating out the massive $U(1)_{X}$ vector supermultiplet. In a general model with canonically normalized matter fields and a dilaton, a similar argument to the one described earlier reveals that

$$
\left\langle D_{X}\right\rangle=\frac{g_{X}^{2}}{M_{X}^{2}}\left(\frac{\delta_{\mathrm{GS}}}{2} K^{\prime \prime \prime}\left|\left\langle F_{S}\right\rangle\right|^{2}-\sum_{i} q_{i}\left|\left\langle F_{i}\right\rangle\right|^{2}-\frac{\delta_{\mathrm{GS}}}{8} k_{X}\left\langle D_{X}\right\rangle^{2}\right),
$$

where now $M_{X}^{2}=g_{X}^{2}\left(\sum_{i} q_{i}^{2}\left|\left\langle\phi_{i}\right\rangle\right|^{2}+\delta_{\mathrm{GS}}^{2} K^{\prime \prime} / 4\right)$. In the model at hand with $\phi_{i}=\varphi, t$, this can be checked to be in precise agreement with Eqs. (14) and (30), to the lowest non-trivial order in $\epsilon$, by plugging in the VEVs and using Eq. (31). 
It is also easy to understand the emergence of the dilatino as the goldstino in the microscopic picture. For a general theory with a Green-Schwarz $U(1)_{X}$ symmetry and chiral superfields $\phi_{i}$ with canonical Kähler potential terms, the fermion mass matrix is given by

$$
M_{\text {fermion }}=\left(\begin{array}{ccc}
k_{X} g_{X}^{2} W^{\prime *} / 2 K^{\prime \prime} & \sqrt{2} g_{X} q_{i} \phi_{i}^{*} & \frac{g_{X}}{\sqrt{2}}\left(\frac{k_{X} D_{X}}{2 \sqrt{K^{\prime \prime}}}-\delta_{\mathrm{GS}} \sqrt{K^{\prime \prime}}\right) \\
\sqrt{2} g_{X} q_{j} \phi_{j}^{*} & \frac{\partial^{2} W}{\partial \phi_{i} \partial \phi_{j}} & \frac{1}{\sqrt{\kappa^{\prime \prime}}} \frac{\partial W^{\prime}}{\partial \phi_{j}} \\
\frac{g_{X}}{\sqrt{2}\left(\frac{k_{X} D_{X}}{2 \sqrt{K^{\prime \prime}}}-\delta_{\mathrm{GS}} \sqrt{K^{\prime \prime}}\right)} & \frac{1}{\sqrt{K^{\prime \prime}}} \frac{\partial W^{\prime}}{\partial \phi_{i}} & \frac{W^{\prime \prime}}{K^{\prime \prime}}-\frac{K^{\prime \prime \prime} W^{\prime}}{K^{\prime \prime 2}}
\end{array}\right)
$$

in the canonically-normalized (gaugino, chiral fermion, dilatino) basis. The goldstino wavefunction in this basis is proportional to

$$
\tilde{G}=\left(\frac{\left\langle D_{X}\right\rangle}{\sqrt{2} g_{X}},\left\langle F_{i}\right\rangle, \sqrt{K^{\prime \prime}}\left\langle F_{s}\right\rangle\right) .
$$

The first row of $M_{\text {fermion }}$ annihilates $\tilde{G}$ by virtue of the gauge invariance condition

$$
\sum_{i} q_{i} \phi_{i} \frac{\partial W}{\partial \phi_{i}}-\frac{\delta_{\mathrm{GS}}}{2} W^{\prime}=0,
$$

while the second and third rows annihilate $\tilde{G}$ by the minimization conditions $\partial V / \partial \phi_{i}=0$ and $V^{\prime}=0$ respectively. Now, we can specialize to the model studied above. Looking only at the lowest order contributions in $\epsilon$, one finds that in the basis $\left(\lambda_{X} / g_{X}, \psi_{t}, \psi_{\varphi}, \psi_{S} / \sqrt{K^{\prime \prime}}\right)$, the goldstino wavefunction is proportional to $\left(0,0, \sqrt{K^{\prime \prime} / K^{\prime}}, 1\right)$. (The zeros actually correspond to terms suppressed by $\epsilon$ and $\sqrt{\epsilon}$.) So in the scenario for dilaton stabilization discussed above, the goldstino is mainly dilatino with a small admixture of the fermionic component of $\varphi$.

In the preceding discussion we have been using a global supersymmetry picture. Including supergravity effects causes the gravitino to obtain a mass by absorbing the goldstino, but does not alter the essential features of the supersymmetry breaking pattern. In particular, including the minimal supergravity terms in the scalar potential does not affect the ratio $\left\langle D_{X}\right\rangle /\left\langle\left.\left\langle F_{S}\right\rangle\right|^{2}\right.$ to leading order in $\delta_{\mathrm{GS}}$.

Let us conclude by noting some phenomenological implications of this analysis. Previously, it was thought that models of dynamical supersymmetry breaking in the presence of an anomalous $U(1)$ featured very small gaugino masses and scalar squared masses dominated by the $D$-term VEV. However, the picture that now emerges is similar to that of a moduli-dominated scenario [21], but with small $D$-term corrections. In the theory below $M_{X}$, assuming a canonical gauge kinetic function (for a possible rationale for this, see [17]) the MSSM gauginos will obtain masses

$$
m_{\lambda}=\frac{\left\langle F_{S}\right\rangle}{\left\langle S+S^{*}\right\rangle} .
$$

Each of the MSSM scalars with $U(1)_{X}$ charge $q_{i}$ receives

$$
m_{\phi_{i}}^{2}=\frac{1}{3} K^{\prime \prime}\left|\left\langle F_{S}\right\rangle\right|^{2}-q_{i}\left\langle D_{X}\right\rangle+\ldots
$$

where the first term represents the usual minimal contribution of the $F$-term of the dilaton, the second is the anomalous $U(1)_{X}$ contribution, and the ellipses refer to other contributions to the soft masses coming from 
higher order Kähler potential couplings between $\phi_{i}$ and $S$, and from contributions due to $F$-terms of other moduli. It is important to remember that such contributions can be comparable to the terms shown explicitly, and need not have any special flavor structure. It follows from Eqs. (28) and (29) that the contributions to the soft masses from $\left\langle F_{S}\right\rangle$ and $\left\langle D_{X}\right\rangle$ are both proportional to $\epsilon \hat{m}$, and so can be made comparable to the electroweak scale by a natural choice of the dynamical scale $\Lambda$. Note that $\left\langle D_{X}\right\rangle$ turns out to be negative in our conventions, so that the $D$-term contributions to MSSM scalar squared masses are positive for $q_{i}>0$. We have found that the $D$-term contributions to scalar masses are likely to be parametrically suppressed by $\delta_{\mathrm{GS}}$ compared to the $F$-term contributions to the gaugino masses (note that $\langle S\rangle$ is typically of order 2 or so) in models where the dilaton is stabilized by large corrections to $K$. If the $F$-term contribution to the scalars are suppressed, it is possible that the $D$-term contributions dominate the tree-level scalar masses. However, renormalization group running yields large flavor-independent positive contributions to the scalar (mass) ${ }^{2}$ proportional to $m_{\lambda}^{2}$, so that the physical masses of squarks and sleptons are again not dominated by the $D$ term.

Since $D_{X}$ is not the dominant source of supersymmetry breaking, we cannot use the anomalous $U(1)$ as a controllable handle on the soft masses. If these theories are to have any hope of being realistic, we must assume that (a) none of the other moduli acquire large $F$-component VEVs and (b) the contributions to scalar masses due to $\left\langle F_{S}\right\rangle \neq 0$ are flavor-blind. Even with these assumptions, it is a quite model-dependent question whether these theories can be consistent with present phenomenological constraints on flavor violation. If the $U(1)_{X}$ charges are family-independent, as in the models in [5,11], then we expect that the $\left\langle D_{X}\right\rangle$ contributions are harmless for flavor-violation even though they are not universal; this makes them particularly interesting for future sparticle spectroscopy. (Of course, other family-dependent $D$-terms present in such models might very well still be dangerous.) On the other hand, in models where the $U(1)_{X}$ symmetry is family-independent, there is a quite serious flavor-violation problem unless the $D$-term contributions to the down squark and slepton squared masses happen to be aligned with the corresponding fermion Yukawa couplings. The presence of larger universal $F_{S}$-term contributions may well ameliorate this problem, and a rough estimate shows that the relative suppression of the $D$-term contributions $\sim \delta_{\mathrm{GS}} \sim 10^{-2}-10^{-3}$ may just be sufficient to explain the absence of flavor-changing neutral currents (for $1 \mathrm{TeV}$ squarks).

If low energy supersymmetry does have something to do with nature, the flavor problem is surely an important clue as to how supersymmetry is broken. If the breaking is at a high scale, one might have hoped that $D$-term breaking with an anomalous $U(1)$ could help resolve this problem. In theories where the $U(1)$ merely serves as a "messenger" of supersymmetry breaking, this could indeed happen, although the gaugino masses tend to be very light and the sfermion spectrum has fine-tuning problems. In theories where the anomalous $U(1)$ dynamics is involved in supersymmetry breaking, we have learned that contrary to the naive expectation, the $D$-term contributions to soft terms in the low energy theory do not, in fact, dominate over Planck scale contributions. Therefore theories of this sort are still subject to potentially dangerous flavor-violating effects from non-minimal contributions to the Kähler potential which involve both $S$ (and the moduli) and the light fields. If we assume that such large flavor-violations are absent, however, anomalous $U(1)$ theories can still be useful for generating the fermion mass hierarchy while evading flavor-changing constraints. There is another positive aspect of our observations. It is usually asserted that in these models the gauginos tend to be very light; this is now seen to be not the case.

In the introduction, we listed five mechanisms for resolving the flavor problems of supersymmetric theories. In this paper we have asked in what sense the fifth, supersymmetry breaking through $D$-terms, is special. We have argued that one should think about this mechanism by integrating out the massive vector field(s). If the mechanism is to be effective, it is crucial that the resulting terms dominate, i.e. that the vector masses be small compared to, e.g. the Planck or string scale. In such a case, soft breaking masses will be controlled by the $U(1)$ charges of the fields - this is the real significance of $D$ term breaking. But we have seen that in theories where the dilaton plays the dominant role in supersymmetry breaking, the couplings of the dilaton to the vector fields are suppressed. In theories such as the $(3,2)$ model, when coupled to the $U(1)$, the $D$ term can dominate the scalar soft breakings, but gaugino masses and the $\mu$ term will be difficult to explain. 


\section{Acknowledgements}

N.A.H. would like to thanks Lance Dixon for useful discussions, and M.D. thanks Yossi Nir for several comments. This work was supported in part by the US Department of Energy.

\section{References}

[1] P. Binétruy, E.A. Dudas, Phys. Lett. B 389 (1996) 503.

[2] G. Dvali, A. Pomarol, Phys. Rev. Lett. 77 (1996) 3728; Anomalous U(1), Gauge-Mediated Supersymmetry Breaking and Higgs as Pseudo-Goldstone Bosons, hep-ph/9708364.

[3] M. Green, J. Schwarz, Phys. Lett. B 149 (1984) 117.

[4] L.E. Ibáñez, G.G. Ross, Phys. Lett. B 332 (1994) 100.

[5] P. Binétruy, P. Ramond, Phys. Lett. B 350 (1995) 49; P. Binétruy, S. Lavignac, P. Ramond, Nucl. Phys. B 477 (1996) 353; P. Binétruy, N. Irges, S. Lavignac, P. Ramond, Phys. Lett. B 403 (1997) 38; J.K. Elwood, N. Irges, P. Ramond, Phys. Lett. B 413 (1997) 322; N. Irges, S. Lavignac, P. Ramond, Predictions from an Anomalous U(1) Model of Yukawa Hierarchies, hep-ph/9802334.

[6] V. Jain, R. Shrock, Phys. Lett. B 352 (1995) 83.

[7] E. Dudas, S. Pokorski, C.A. Savoy, Phys. Lett. B 356 (1995) 45; E. Dudas, C. Grojean, S. Pokorski, C.A. Savoy, Nucl. Phys. B 481 (1996) 85.

[8] E.J. Chun, A. Lukas, Phys. Lett. B 387 (1996) 99; K. Choi, E.J. Choi, H. Kim, hep-ph/9611293.

[9] R.N. Mohapatra, A. Riotto, Phys. Rev. D 55 (1997) 1137; D 55 (1997) 4262.

[10] A.E. Nelson, D. Wright, Horizontal, anomalous $U(1)$ symmetry for the more minimal supersymmetric standard model, hep-ph/9702359.

[11] A.E. Faraggi, J.C. Pati, A Family-Universal Anomalous U(1) in String Models as the Origin of Supersymmetry Breaking and Squark Degeneracy, hep-ph/9712516.

[12] I. Affleck, M. Dine, N. Seiberg, Nucl. Phys. B 241 (1984) 493; B 256 (1985) 557.

[13] S. Dimopoulos, G.F. Giudice, Phys. Lett. B 357 (1995) 573.

[14] N. Arkani-Hamed, H. Murayama, Phys. Rev. D 56 (1997) 6733.

[15] N.V. Krasnikov, Phys. Lett. B 193 (1996) 103; J.A. Casas, Z. Lalak, C. Muñoz, G.G. Ross, Nucl. Phys. B 347 (1990) 243; B. de Carlos, J.A. Casas, C. Muñoz, Nucl. Phys. B 399 (1993) 623.

[16] S.H. Shenker, in: Proceedings of the Cargese School on Random Surfaces, Quantum Gravity and Strings, Cargese, France, 1990.

[17] T. Banks, M. Dine, Phys. Rev. D 53 (1996) 5790.

[18] P. Binétruy, M.K. Gaillard, Y.-Y. Wu, Nucl. Phys. B 481 (1996) 109; B 493 (1997) 27; Phys. Lett. B 412 (1997) 228.

[19] J.A. Casas, Phys. Lett. B 384 (1996) 103.

[20] T. Barreiro, B. de Carlos, E.J. Copeland, On non-perturbative corrections to the Kähler potential, hep-ph/9712443.

[21] V. Kaplunovsky, J. Louis, Phys. Lett. B 306 (1993) 269; R. Barbieri, J. Louis, M. Moretti, Phys. Lett. B 312 (1993) 451; A. Brignole, L.E. Ibáñez, C. Muñoz, Nucl. Phys. B 422 (1994) 125; B 436 (1995) 747 (E). 